\title{
Nouveau regard sur le comportement de l'as- perge (Asparagus officinalis L.) multipliée in vitro
}

\author{
Claire DORÉ \\ avec la collaboration technique de Florence Charlot \& Jean-Charles LESCURE: \\ I.N.R.A., Station de Génétique et d'Amélioration des Plantes, route de Saint-Cyr, F 78026 Versailles Cedex
}

RÉSUMÉ

La possibilité de multiplier végétativement in vitro l'asperge a permis notamment de cloner les parents des variétés hybrides de clones.

L'obtention d'une griffe fonctionnelle dès le stade in vitro est un facteur essentiel de réussite de la micropropagation.

Des améliorations permettant de maîtriser la méthode de clonage sont proposées ici. Une série de microbouturages est nécessaire pour induire ultérieurement de nombreux bourgeons qu'il est possible de fragmenter comme des griffes.

Les explants présentent, au cours de la culture, une importante évolution de leurs capacités morphogènes qu'il a été possible de confirmer au niveau biochimique.

Le comportement de l'asperge présente de nombreuses similitudes avec celui des arbres in vitro. Ce comportement pourrait être attribué au caractère pérenne de ces espèces.

Ce « rapprochement » permet d'expliquer les difficultés rencontrées chez l'asperge et peut offrir des perspectives intéressantes en matière de multiplication in vitro chez les espèces pérennes.

Mots clés additionnels: Multiplication végétative, culture in vitro, griffe, arbre, marqueurs biochimiques, rajeunissement, pérennité.

\begin{abstract}
Asparagus cloning revisited.
In vitro vegetative multiplication of asparagus has especially allowed cloning of the parents of clonal hybrid varieties. Obtaining a functional crown at the in vitro stage is essential for successful micropropagation. Various improvements to the method are proposed here. Successive microcuttings were found necessary to induce numerous shoots which can be separated as crowns. During culture, explants changed considerably in morphogenic ability, which could be confirmed at the biochemical level. The behaviour of asparagus was very similar in vitro to that of trees, presumably because it is a perennial crop. This parallel helps to explain the difficulties encountered in asparagus micropropagation and could lead to interesting future prospects in improving cloning of perennial crops.
\end{abstract}

Additional key words : Vegetative multiplication, in vitro culture, crown, biochemical markers, tree, rejuvenation, perennity.

\section{INTRODUCTION}

L'asperge (Asparagus officinalis L.), de la famille des Liliacées, est une espèce diöque et pérenne dont la multiplication végétative en conditions horticoles est possible mais limitée. Chez cette monocotylédone, la pérennité de la plante est assurée par une griffe comportant un rhizome, et constituée de bourgeons souterrains et de racines de réserve réparties en plusieurs pôles.

Au printemps de chaque année, des turions, qui sont les asperges destinées à la consommation, se dévelop- pent à partir des bourgeons souterrains pour donner des tiges ramifiées. Ces tiges portent des cladodes, ayant eux-mêmes une structure de tige, tandis que les feuilles sont réduites à des écailles situées le long des turions.

La croissance du rhizome et l'émission des tiges sont, en particulier, sous la dépendance de la dominance apicale et de la température.

La floraison d'une plante n'est induite qu'environ un an après son semis, la pleine exploitation d'une plantation ne commençant que quatre ans après le semis (THÉVENIN, 1967b). 
Les travaux de sélection entrepris chez cette espèce pour augmenter le rendement ont permis de repérer des combinaisons hybrides très performantes entre 2 parents hétérozygotes (TIIÉVENIN, 1967a, 1971). L.'intérêt de ces hybrides simples est tel qu'il apparaît important de disposer d'un moyen pour cloner les deux parents.

Le développement des techniques de culture de l'asperge in vitro a suscité dans le monde entier de nombreux travaux (DORÉ, 1975), dont l'un des objectifs est, en particulier, la multiplication conforme. Deux méthodes ont été employées: la culture d’apex (ou de méristèmes) et le microbouturage.

Par culture d'apex ou de méristèmes, l'effectif obtenu résulte du nombre élevé de méristèmes présents à l'extrémité d'un turion et qui, mis en culture isolément, vont se développer, pour donner chacun une plante. Les plantes produites comportent des liges et des racines mais l'absence de griffe, in vitro, entraine des difficultés au moment du repiquage en terre. En conditions horticoles, seules les plantes ayant initié une griffe poursuivent leur développement. MurashigE et al. (1972), HASEGAWA et al. (1973), TENDILLE \& LECER F (1974), DORÉ (1975), YANG \& ClORE (1976), ont utilisé les nombreux méristèmes présents sous les écailles à l'extrémité du turion, tandis que RIVIÉRE \& MULleER (1974) ont utilisé des bourgeons souterrains de la griffe.

Dans le cas du microbouturage, ce sont les nouds situés au niveau des écailles du turion qui sont utilisćs et l'effectif obtenu résulte du fractionnement répété au cours des repiquages successifs, des tiges développées à partir des nouds. Une griffe fonctionnelle est ensuite induite in vitro ce qui permet un transfert en terre sans difficulté (DORÉ, 1974 et 1975, YANG \& CLORE, 1973 et 1974, THEVENIN \& DORÉ, 1976).

L'application de ces méthodes a permis de réaliser notamment le clonage des géniteurs hétérozygotes, el un nouveau type de variéte hybride, l'hybride de clones, a pu être créé et diffusé : Aneto, Desto, Cito et Stéline en France (DORÉ, 1977), UC 157 et Jersey Giant aux Etats-Unis (BENSON \& TAKATORI, 1980, ELLISON, 1984), et Diego en Italie (FALAVIGNA et al., 1982).

Bien que ces méthodes aient permis la diffusion des variétés hybrides de clones, des difficultés importantes ont été rencontrées par tous les sélectionneurs: le clonage à grande échelle des 2 individus parents de chaque hybride, indispensable pour la diffusion commerciale de l'hybride, s'est heurté en effet à divers problèmes: délais pour atteindre in vitro l'effectif souhaité, faible taux d'enracinement, difficultés d'acclimatation du matériel aux conditions horticoles, etc...

Ces difficultés se sont traduites, dans tous les pays concernés, par un retard à la "sortie commerciale " des hybrides de clones et par une limitation durable des disponibilités en semences.

C'est pourquoi des recherches ont été poursuivies pour parvenir à une meilleure maîtrise de l'obtention des clones.

La pratique des 2 méthodes de clonage nous ayant démontré que l'induction de la griffe in vitro était la clé de la multiplication végétative, nous avons cherché à améliorer les performances du microbouturage et de très nombreuses expériences ont été effectuées sur divers matériels végétaux.
La première partie de cet article fera part des observations qui nous ont conduite à établir une nouvelle méthode assurant une production régulière de plantes et du protocole de cette méthode. La deuxième partie sera consacrée aux premiers éléments de l'analyse des différentes étapes du clonage qu'il est dès lors possible d'envisager.

\section{MATÉRIEL VÉGÉTAL}

Divers matéricls correspondant à plus de vingt génotypes ont été utilisés:

- des jeunes tiges régénérées à partir de cals issus de culture d'anthères (DORE, 1974) ou de cellules isolées (JULLIEN, 1973).

- des turions prélevés en serre sur des plantes âgées de 5 à 10 ans (géniteurs hétérozygotes, plantes issues de culture d'anthères) ;

- des turions prélevés en chambre climatisée sur des plantes issues des deux matériels précédents, après 6 mois de culture en terre.

\section{MISE AU POINT \\ DUUNE NOUVELLE MÉTHODE DE CLONAGE}

\section{A. Méthode préalable}

Les conditions de culture et la méthode de microbouturage ayant déjà été décrits (DORÉ, 1975), nous nous bornerons ici à résumer cette méthode qui a servi de point de départ à nos travaux ultérieurs.

Le microbouturage consiste à prélever des fragments de tige comportant un nœud et à les placer sur un milieu $\mathrm{T}_{0}$ sans substance de croissance. Des repiquages successifs de ces fragments après suppression des tiges nouvellement formées, conduisent à l'initiation et au développement d'une griffe in vitro. Chaque tige peut être elle-même découpée en fragments pour augmenter l'effectif disponible. Le transfert en terre est effectué lorsque les racines secondaires sont apparues.

\section{B. Observations}

1. Nous avons pu confirmer certaines observations effectuées sur quelques génotypes et déjà rapportées en 1975 : elles peuvent être généralisées à l'ensemble des génotypes étudiés :

\section{a) Importance du stade de végétation du materiel de départ}

Seuls les nœuds situés sur des tiges prélevées avant la phase de ramification comportent des bourgeons latents aptes à produire une ou plusieurs tiges en culture in vitro.

\section{b) Existence d'un gradient le long de la tige}

Le diamètre du turion n'a pas d'influence sur le « devenir » de la culture, mais les nœuds situés près de la 
base du turion réagissent toujours mieux en particulier chez les plantes âgées. Ces résultats sont à rapprocher, d'une part, des observations de YANG \& CLORE (1973) qui obtenaient les meilleurs enracinements et les plantes les plus vigoureuses à partir d'explants de la base, et d'autre part des travaux de RivièRE \& MulLER (1974) qui s'adressaient à des bourgeons souterrains, donc proches des racines.

\section{c) Importance de la dominance apicale}

L'apparition de la griffe in vitro est liée à la levée de la dominance apicale.

De simples transferts du matériel végétal sur un milieu « frais » ne permettent pas d'obtenir l'induction d'une griffe; il est nécessaire de supprimer systématiquement les tiges nouvellement formées pour permettre l'apparition de celle-ci.

Par ailleurs, la succession de périodes longues entre deux repiquages a tendance à provoquer une entrée en dormance du matériel.

\section{d) Importance de la structure de la racine obtenue}

Pour faciliter l'acclimatation en conditions horticoles, la griffe doit être présente et comporter des racines de réserve bien développées et des racines secondaires. Ces dernières assurant l'absorption chez l'asperge, leur présence au moment du repiquage en terre est essentielle, ce qui confère un avantage à l'utilisation d'un milieu liquide avant le transfert en terre.

\section{Nous avons en outre noté :}

\section{a) L'effet de l'âge physiologique du matériel de départ}

La pratique de la culture d'anthères in vitro (DORÉ, 1974 et 1989) nous a conduite à remarquer une évolution très nette des possibilités de multiplication des tiges régénérées à partir de cals d'origine androgénétique. Ainsi pour les tiges qui proviennent directement de la régénération des cals, deux ou trois repiquages seulement sont nécessaires pour obtenir une multiplication active qui s'accompagne d'un enracinement de type griffe, et il est en outre possible de constituer rapidement un clone disponible pour la sélection (THEVENIN \& DORÉ, 1976). Après plusieurs années de culture en conditions horticoles, ces capacités de multiplication ont disparu et ce n'est qu'après de nombreux repiquages (environ six) que des griffes peuvent être induites à nouveau in vitro.

Nous avons pu confirmer ces observations avec du matériel provenant de culture de cellules isolées que nous a fourni $M$. JuLliEN (CNRS, Gif-sur-Yvette). Pour un même génotype, les possibilités de multiplication active et d'enracinement rapide diminuent donc, à mesure que l'âge de la plante sur laquelle on prélève le matériel, évolue.

b) L'importance de l'équilibre des substances de croissance

La présence de cytokinine (benzyladénine ou kinétine) dans le milieu de culture accélère la formation de la griffe, mais n'est pas indispensable. Un développement harmonieux des tiges, annoncé par la présence d'écailles le long des tiges et l'absence de cladodes au niveau de la base, est indispensable pour assurer une émission régulière de bourgeons ayant un intérêt potentiel pour l'initiation de la griffe.

Seuls quelques équilibres de substances de croissance le permettent. Des modifications mêmes légères de ces équilibres entraînent une perturbation de la croissance des bourgeons de la future griffe et la formation de celle-ci est alors, le plus souvent, remise en cause de manière irréversible. Dans nos conditions de culture, c'est un équilibre composé de $0,1 \mathrm{mg} / 1$ de benzyladénine (B.A.) et de $0,01 \mathrm{mg} / 1$ d'acide naphtalène acétique (A.N.A.) qui est le plus favorable. L'utilisation de deux cytokinines en association (B.A. et kinétine) est également un facteur favorisant l'émission des bourgeons (ROSSI \& DE PAOLI, 1986).

\section{c) L'évolution des capacités organogènes au cours du temps}

Dans les premiers stades de culture, les explants et les microboutures utilisés qui émettent, à leur base, pendant chaque culture, de nouveaux bourgeons, ne se prêteraient pas sans risque à une division en raison de leur faible nombre et de la disposition relative de ces derniers. Au contraire lorsqu'on a effectué un certain nombre de repiquages dont le nombre nécessaire augmente avec l'âge de la plante de départ, le comportement du matériel se modifie brusquement par l'apparition de bourgeons trapus et une accélération de la croissance globale du fragment (explant comme microbouture). Le nombre de bourgeons et leur disposition permettent alors une fragmentation. Il est possible de réduire la durée entre deux repiquages et on assiste alors à une sorte d'《 emballement » du processus de multiplication. A ce stade, l'addition dans le milieu d'une substance antigibbérellique, l'ancymidol $\left(^{*}\right)$ à la dose de $1,3 \mathrm{mg} / 1$ selon CHIN (1982), favorise une production intensive de bourgeons accompagnée de l'apparition de nombreuses racines de réserve.

Par contre, l'application de cette substance antigibbérellique aux stades précédents, mise en culture ou premiers repiquages, est tout à fait défavorable. La réceptivité du matériel vis-à-vis de ce produit évolue donc au cours du temps.

\section{Nouvelle méthode de clonage}

L'ensemble de ces observations nous conduit à proposer la méthode suivante :

\section{Protocole}

Le microbouturage est pratiqué à partir des nœuds des écailles le long du turion ou de la tige, et les explants sont placés in vitro sur un milieu $\mathrm{Y}_{12}$ contenant le milieu

(*) Ce produit nous a été aimablement fourni par ELI LILLY FRANCE. 
de base de Murashige \& SKoOg (1962) additionné de $0,01 \mathrm{mg} / 1 \mathrm{~d}$ 'A.N.A. et de $0,1 \mathrm{mg} / 1$ de B.A..

Des repiquages successifs du nœud sont pratiqués toutes les 4 à 6 semaines en supprimant systématiquement la majeure partie de chacune des tiges formées.

Les tiges supprimées au cours des premiers repiquages sont elles-mêmes bouturées et les microboutures placées sur le milieu $Y_{12}$ pour augmenter l'effectif des explants. Après un certain nombre de repiquages (de deux à six selon l'âge physiologique du matériel de départ), les explants et les microboutures émettent à leur base de nombreux bourgeons qu'il est possible de fragmenter toutes les trois semaines pour atteindre l'objectif désiré. Dès ce stade, de l'ancymidol $(1,3 \mathrm{mg} / \mathrm{l})$ peut être ajouté dans le milieu de culture. Lorsque l'effectif est atteint, de l'ancymidol $(1.3 \mathrm{mg} / \mathrm{l})$ et du saccharose à la dose de $60 \mathrm{~g} / \mathrm{l}$, selon DESJARDINS et al. (1986), sont ajoutés, pour favoriser l'enracinement. Un ultime repiquage, avant le transfert en conditions horticoles, sur le milieu liquide $\mathrm{T}_{0}$ est favorable pour lever l'inhibition du développement des tiges provoquée par l'ancymidol.

\section{Conditions de culture}

Les fragments végétaux sont disposés à raison de 10 par bocal, dans des bocaux en verre de $3,75 \mathrm{cl}$ comportant un couvercle en matière plastique, et placés dans une salle de culture (thermopériode $18-23^{\circ} \mathrm{C}$, photopériode de 16 heures, éclairement de $12 \mathrm{~W} / \mathrm{m}^{2}$ ).

Lors du transfert en conditions horticoles, les plantes sont placées dans un mélange stérile de terreau, sable et tourbe $(1: 1: 1)$ dans une "mini-serre" qui est ouverte progressivement pour assurer l'endurcissement des plantes. L'ensemble est placé en chambre climatisée (thermopériode de $12-28^{\circ} \mathrm{C}$, photopériode de 16 heures, éclairement de $50 \mathrm{~W} / \mathrm{m}^{2}$, humidité relative de 60 p. 100) durant 1 à 3 mois, avant passage en serre chauffée et éclairée.

\section{Conservation in vitro}

Le matériel végétal peut être conservé pendant 6 mois sans acune intervention sous forme de fragments (explants ou microboutures) au stade de la production de bourgeons ou sous forme de plantes racinées, soit dans les conditions de la salle de culture décrites cidessus, soit dans une chambre à $7{ }^{\circ} \mathrm{C}$ avec une photopériode de 10 heures et un éclairement de $6 \mathrm{~W} / \mathrm{m}^{2}$.

\section{LES DIFFÉRENTES ÉTAPES DU CLONAGE PREMIERS ÉLÉMENTS D'ANALYSE}

La mise en æuvre du protocole ci-dessus qui conduit à une production régulière de plantes a été réalisée avec de nombreux génotypes.

Dans tous les cas, les résultats obtenus conduisent à distinguer schématiquement trois phases dans la courbe de production en relation avec l'importante évolution physiologique du matériel végétal au cours du temps (fig. 1) :

\section{Une phase préliminaire}

Pendant cette phase le matériel évolue lentement et nécessite des repiquages successifs avec suppression des

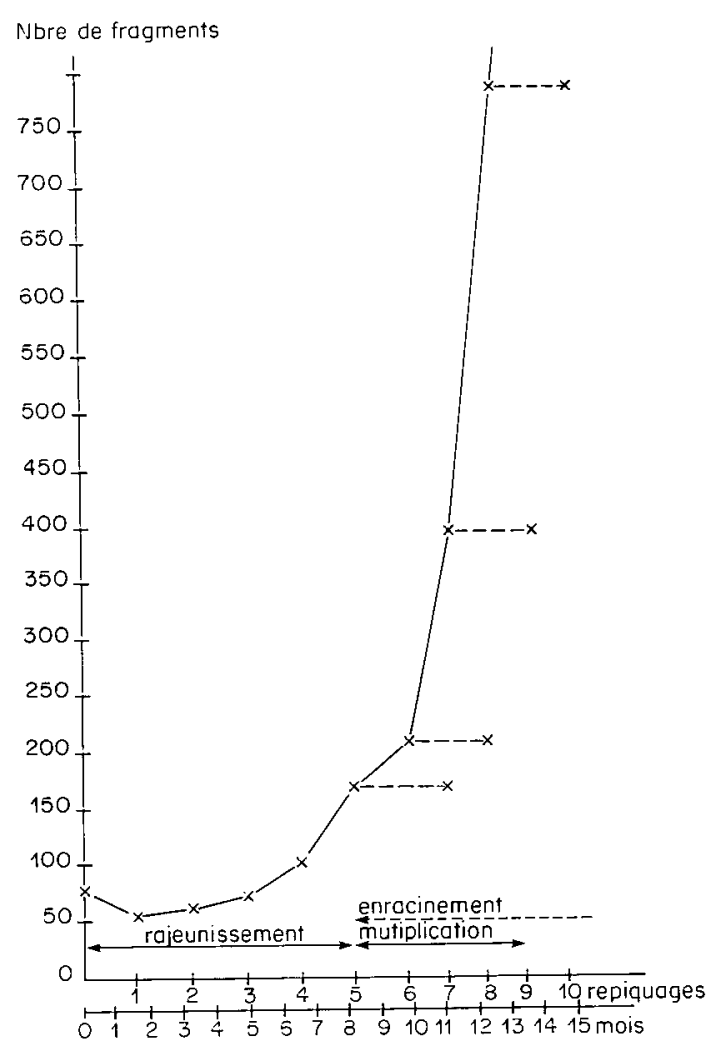

Figure 1

Profil schématique de la courbe de clonage in vitro de l'asperge. Schematic outline of the cloning curve of asparagus in vitro.

tiges nouvellement formées. Aucune racine n'apparaît à ce stade et il n'y a pratiquement pas de multiplication. La longueur de cette phase augmente avec l'âge physiologique du végétal ayant fourni l'explant d'origine. A ce stade, l'addition d'ancymidol n'est pas favorable.

Lors des premiers repiquages, le microbouturage des tiges formées permet d'accroître sensiblement l'effectif global.

\section{Une phase de multiplication}

Le début de cette phase est marqué par une évolution des fragments : leur activité prolifératrice s'accélère et ils forment des touffes de bourgeons structurés en griffes. Pendant cette période, l'induction de nombreuses griffes est favorisée par l'addition d'ancymidol dans le milieu de culture. Le taux de multiplication est de l'ordre de 2 , à chaque repiquage.

\section{Une phase d'enracinement}

Celle-ci peut être enclenchée à tout moment dès que la deuxième phase est atteinte. Elle permet d'obtenir le complet développement des plantes avant passage en conditions horticoles.

Au cours de ces trois phases successives qui caractérisent le clonage in vitro de l'asperge, l'effectif progresse d'abord lentement, puis, peut doubler à chaque repiquage dès que le processus de multiplication est installé. La forme de la courbe de multiplication est la même pour les explants et les microboutures. Seule l'origine est décalée dans le cas de la courbe des microboutures, 
comme le montre l'exemple du génotype femelle $\mathrm{n}^{\circ} 2$ (fig. 2).

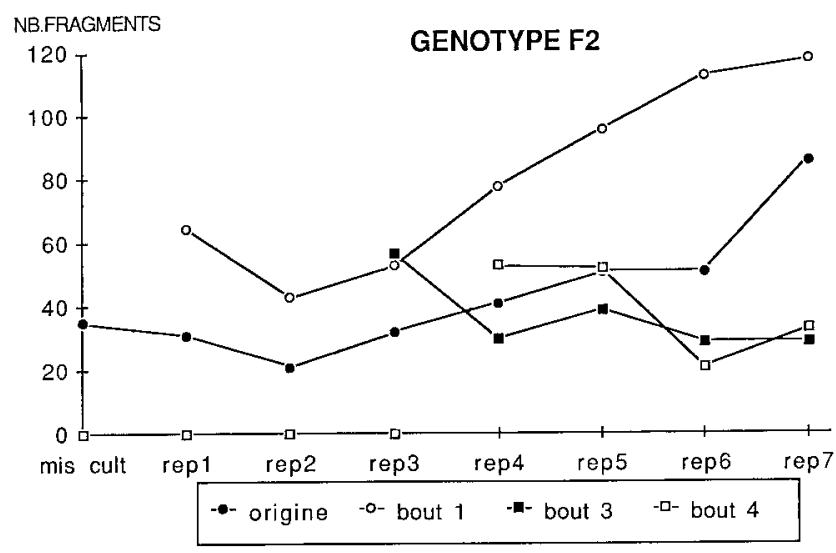

Figure 2

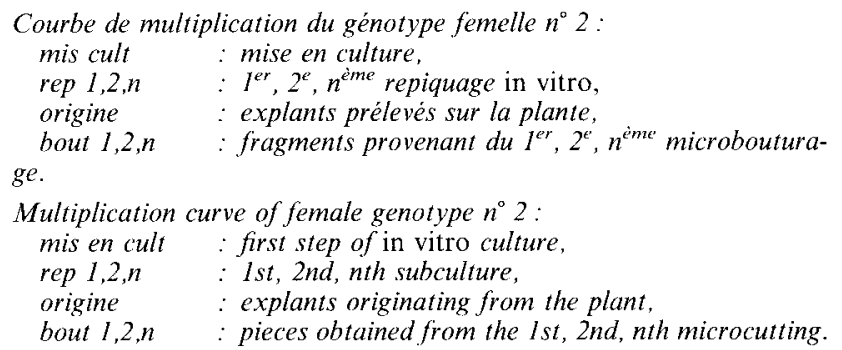

Nous avons cherché à savoir si le passage d'une phase à l'autre s'accompagnait dans le matériel végétal de modifications biochimiques décelables. Une étude préliminaire, sur un génotype, a été réalisée en collaboration avec le Laboratoire d'Etude du Métabolisme et de Nutrition des Plantes de l'INRA à Versailles (V. FIALA \& J.P. BoUTIN). Une analyse par chromatographie liquide d'échange d'ions (FIALA \& JoliveT, 1982), a permis de préciser la composition en acides aminés totaux, en amines et en sucres solubles de la base des nœuds conservée au cours des repiquages successifs.

Les premières analyses font apparaître une évolution régulière et sensible de la quantité de certains composés. On note en particulier que la quantité d'acides aminés libres totaux ne cesse de croître au cours de la culture : cinq d'entre eux dont la citrulline, l'arginine et la proline augmentent de façon notable. D'autre part, on constate que les teneurs en putrescine et spermidine, polyamines présentes en quantité non négligeable chez l'asperge, augmentent de manière significative, pendant la production intense de bourgeons; ainsi pour la putrescine, cette teneur passe de 91 à $344 \mathrm{n}$ moles/g de matière fraîche (FIALA \& BOUTIN, communications personnelles).

Enfin, l'étude quantitative que nous avons plus particulièrement menée à partir de 2 géniteurs femelles et 3 géniteurs mâles nous a permis d'apprécier l'influence du génotype sur le déroulement du clonage. Cette influence, qui est d'ailleurs une caractéristique constante chez l'asperge (THÉVENIN \& DORÉ, 1976) se manifeste ici à tous les niveaux : d'un génotype à l'autre, l'aptitude variable au clonage se traduit par des réactions diverses, dès la première culture, au niveau du nombre d'explants initiant des tiges, du nombre et du diamètre des bourgeons formés, du nombre de microboutures, du nombre de fragments possibles, etc... Si la forme de la courbe de production est toujours la même quel que soit le génotype, il n'en est pas de même pour la pente qui est sensiblement différente selon les génotypes (fig. 3). Les variations observées peuvent être également importantes en ce qui concerne la fréquence d'enracinement $(27$ à 95 p. 100 avec une fréquence moyenne de 70 p. 100).

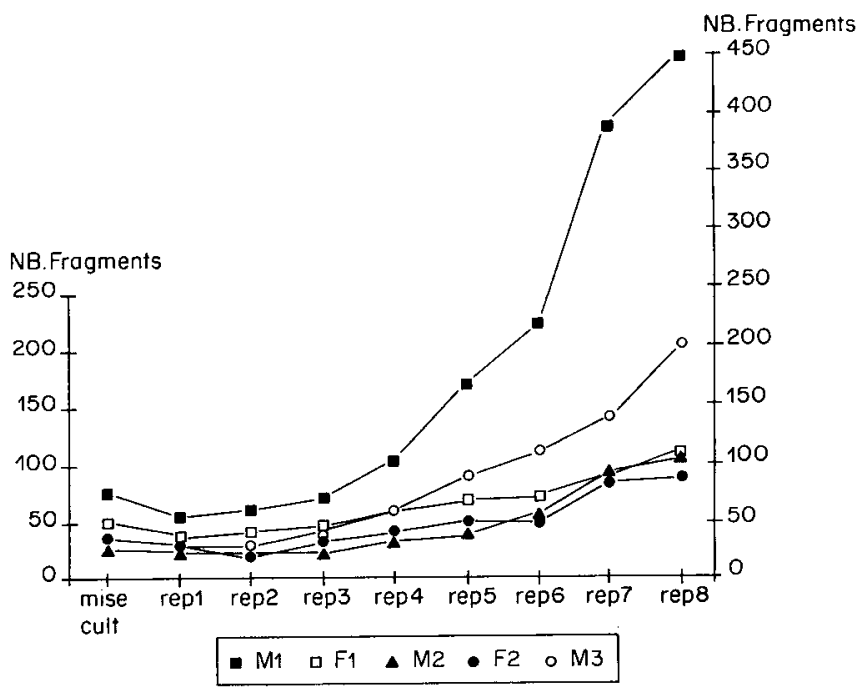

Figure 3

Courbe de multiplication de différents génotypes d'asperge: 2 géniteurs femelles $\left(F_{1}\right.$ et $\left.F_{2}\right)$ et 3 géniteurs mâles $\left(M_{1}, M_{2}\right.$ et $\left.M_{3}\right)$. Multiplication curve of different asparagus genotypes: two female genitors $\left(F_{1}\right.$ et $\left.F_{2}\right)$ and three male genitors $\left(M_{1}, M_{2}\right.$ and $\left.M_{3}\right)$.

Cette variabilité de comportement se rencontre aussi bien chez les plantes femelles que chez les mâles: l'aptitude au clonage n'est donc pas liée au sexe mais au génotype.

\section{DISCUSSION}

Les résultats actuels permettent de considérer que l'on dispose désormais d'une méthode fiable de clonage et de conservation in vitro chez l'asperge. Ceci correspond à un progrès important et a permis d'envisager une première analyse.

Cette succession de microbouturages et de fragmentations in vitro, qui constitue notre méthode actuelle de clonage, présente des similitudes certaines avec les méthodes utilisées pour multiplier les arbres et les arbustes (CORNU, 1977 ; MULLINS et al., 1979 ; FRANCLET, 1980 ; BONGA, 1982).

En effet, la micropropagation des arbres adultes commence assez souvent par une phase de rajeunissement pendant laquelle l'effectif n'augmente quasiment pas. Cette phase qui fait appel à diverses pratiques culturales, permet d'obtenir à nouveau un certain état juvénile. Les potentialités liées à cet état se traduisent en particulier par des possibilités de multiplication intense et une aptitude à la rhizogenèse (FRANCLET et al., 1987 ; HACKETT, 1987). 
Comme l'asperge, les arbres et les arbustes sont des espèces pérennes et il est possible que leur comportement particulier vis-à-vis du clonage in vitro soit lié au caractère de pérennité plutôt qu'au caractère ligneux propre aux arbres.

Cette hypothèse vient éclairer les faits d'un regard nouveau. Par exemple :

- la phase préliminaire peut être considérée comme une phase de rajeunissement ;

- les propriétés remarquables des tiges issues de cals s'expliquent par leur caractère juvénile;

- la putrescine et la spermidine, polyamines antisénescentes précurseurs des acides nucléiques, dont la quantité augmente brusquement au début de la phase de mutiplication, apparaissent comme des marqueurs logiques de ce changement.

Les possibilités de conservation du matériel à un stade, où il est déjà en phase de multiplication active permettent de constituer un "pied de cuve"; ceci représente un gain de temps important car le matériel, déjà rajeuni, est toujours disponible pour engager une nouvelle phase de multiplication sans avoir recours à la phase de rajeunissement préalable.

La mise en évidence de marqueurs biochimiques peut s'avérer très intéressante. Des études doivent être poursuivies pour caractériser de façon plus précise, à l'aide de ces marqueurs, les différentes étapes du clonage chez l'asperge et tenter de raccourcir la phase de rajeunissement. Laanalyse quantitative de l’influence des divers composants du milieu de culture devient possible avec de tels marqueurs et permet d'envisager notamment l'étude de l'influence de l'addition dans le milieu de culture de ces polyamines ou de leurs précurseurs (arginine et ornithine).

L'asperge peut constituer un modèle qui pourrait s'avérer fort utile dans le cadre d'études consacréces à la multiplication de certaines autres espèces pérennes. Comparée aux arbres, sa période juvénile est courte, ce qui la rend plus accessible pour des travaux de physiologie.

La caractérisation biochimique du rajeunissement est un objectif important des études conduites actuellement pour améliorer les possibilités de multiplication végétative chez les espèces forestières (JAY-LALLEMAND et al., 1987).

Dans ce contexte, il serait intéressant de savoir si certains arbres et l'asperge comportent les mêmes marqueurs de l'état juvénile.

\section{CONCLUSION}

La multiplication végétative in vitro de l'asperge est désormais bien maîtrisée : la méthode fiable de clonage proposée ici a permis d'établir de nombreux points communs entre le comportement de l'asperge et celui des arbres in vitro.

C"est vraisemblablement à ce caractère pérenne commun à toutes ces espèces qu'est liée la difficulté de leur multiplication végétative.

Outre l'intérêt d'ordre agronomique indéniable de cette méthode de propagation, ce « rapprochement» permel d'établir des perspectives d'études qui devraient concourir efficacement à faire progresser les connaissances dans le domaine de la multiplication in vitro des espèces pérennes.

Resule 18 décembre 1987. Accepte le 14 siptembre 1988.

\section{REMERCIEMENTS}

Lateur remercie Madame L. CORRIOLs pour sa constante collaboration scientifique al ce travail, Messieurs V. FiALA et J. P. BOUTIN pour les analyses biochimiques quils ont réalisées, et Messieur: D. CORNU et C. JAY-LALLEMAND (INRA. Orléans) pour les informations quils nous ont fournies sur les arbres.

\section{RÉFERT:NCES BIBLIOGRAPHIQUES}

Benson B., Takatori F., 1980. Foundalion Asparagus Sesd program and the release of UC 157 parental clones to the seed ind astry. Asparagus Research. 1979-1980. Univer. of Calif., Davis. 25-26.

Bonga J. M., 1982. Vegetative propagation in relation to juven:lity, maturity and rejuvenation. In : Tissue Culmo' in Forestr. BonGA \& DURzAN (cds). M. Nijhofl. 387-412.

Chin C. K., 1982. Promotion of shoot and root formation in asparagus in vitro by ancymidol. Hortscience, 17, 590-591.

Cornu D., 1977. La multiplication végétative du noyer hybride Résultats d'une première campagne. Perspectives d'avenir. Re't. forest franc.. 29, 6. 457-463

Desjardins Y., Tiessen H., Harney P. M., 1986. The effect of sucrose and ancymidol on the in vitro rooting of nodal sections of asparagus. Hortscience, 22, 1, 131-133.

Doré C., 1974. Production de plantes homozygotes mâles et femelles à partir d"anthères d'Asperge. C.R. Acad. Sci. Paris, Ser. D., 278. 2135-2138.

Doré C., 1975. La multiplication clonale de l'Asperge (Asparrgus officinalis L.) par culture in vitro: son utilisation en sélection. Ann. Amélior. Plantes, 25, 2, 201-224.

Doré C., 1977. In vitro techniques as an efficient tool in asparagus breeding. Acta Hortic, 78, 89-93.

Doré C., 1989. Asparagus anther culture. In : Biotechnology in Agriculture and Forestry, vol. 12, Bajaj (ed.), Springer-Verlag (à paraître).
Ellison II., 1984. Release of Jersey Giant and Greenwich. Asparagus Re'search Newst'ter', 2, 1, p. 23

Falavigna A., Casali P., Soresi G., 1982. Diego : first ltalian hybrid of asparagus from heterozygous clones. Genet. agrar.. 162-163.

Fiala V., Jolivet E., 1982. Variations quantitatives en composés azotés el glucidiques de racines d'Asperges mâles el femelles. au cours de leur premicre année de culture. Agronomic, 2, 9, 735-740.

Franclet A., 1980. Rajeunissement et propagation vćgćtative des ligneux. Anh. A.F.O.C.E.L., 11-40.

Franclet A., Boulay M., Bekkaoui F., Fouret Y., Ver SchooreMartouzet B., Walker N., 1987. Rejuvenation. In : Cell and Tissuc Culture in Forestru, vol. 1, Bonga \& Don Durzan (eds), M. Nijhoff, 2.32-248.

Hackett W. P., 1987. Juvenility and maturity. In : Coll and Tissuc Cullure in Forestry, vol. 1. Bonga \& Durzan (eds). M. Nijhoff, 216231

Hasegawa P. M., Murashige T., Takatori F. H., 1973. Propagation of asparagus through apex culture. II. J. am. Soc. hort. Sci., 98, 2, 143-148.

Jay Lallemand C., Cornu D., Macheix J. J., 1987. Caractérisation du rajeunissement du Noyer (Juglams sp.) par une étude spectrophotométrique globale du contenu polyphénolique. Ann. Sci. for.. 44 (3). 303-314. 
Jullien M., 1973. La culture in vitro de cellules séparées du tissu foliaire d'Asperge. C. R. Acad. Sci., Paris, Ser. D, 276. 733-736.

Mullins M. G., Nair Y., Sampet P., 1979. Rejuvenation in virro: induction of juvenile characters in an adult clone of Vitis vinifera $\mathrm{L}$. Am. Bot., 44, 623-627.

Murashige T., Skoog F., 1962. A revised medium for rapid growth and bioassays with tobacco tissuc cultures. Phisiol. Plamt., 15, 473-497.

Murashige T., Shabde M. N., Hasegawa P. M., Takatori F. H., Jones J. B., 1972. Propagation of asparagus through shoot apex culturc. I. Nutrient medium for formation of plantlets. J. Am. Soc. hort. Sic., 97, 158-161.

Riviere S., Muller J. F., 1974. Résultats obtenus par la multiplication végétative in vitro des bourgeons souterrains d"Asparagus officinalis $\mathrm{L}$. C. R. Acat. Sci., Paris, Ser. D, 279, 899-902.

Rossi V., De Paoli G., 1986. Micropropagazione e conservazione in vitro dell' asparago. Agricolinura Riceréca, 63/64, 33-40.

Tendille C., Lecerf M., 1974. La multiplication végétative de l'Asperge (Asparagus officinalis L.). Action de divers facteurs en particulier de la nutrition minérale. sur le développement des méristemes d'Asperge, sur la croissance des plantules issues de ces méristèmes et sur la production de plantes adultes. Amm. Amélior. Plantes. 24, 3, 269-282.

Thevenin L., 1967a. Asperge. Rapporr 1965-1967. Station de Génétique et d’Amélioration des Plantes. 23-29.

Thevenin L., 1967h. Les problèmes damélioraltion chez Asparaguts officinalis L. I. Biologie et Amélioration. Ann. Amélior. Plantess, 17, 1 33-36.

Therenin L., 1971. Asperge. Rapport d'activite' 1968-1971. Station do Gínétique et d'Amélioration des Plantes. $30 \mathrm{p}$.

Thevenin L., Doré C., 1976. L'amélioration de l'Asperge et son atout majeur, lal culture in vitro. Ann. Amólior. Plantes, 26. 4, 655-674.

Yang I. J., Clore W. J., 1973. Rapid vegetative propagation of asparagus through Iiteral bud culture. Hortscicnce', 8. 2, 141-143.

Yang H. J., Clore W. J., 1974. Development of complete plantlets from moderatcly vigorous shoots of stock plants of Asparagus in vitur. Hortscicince, 9, 2, 138-140.

Yang H. J., Clore W. J., 1976. Obtaining virus-free plants of A. officinalis by culturing shoot tips and apical meristems. Hortscince $11,5,474-475$ 